\title{
Machine Vision based Agricultural Weed Detection and Smart Herbicide Spraying
}

\author{
S. Mohan Raj* and V. Kavitha \\ Department of Electronics and Communication Engineering, M. Kumarasamy College of Engineering, \\ Karur - 639113, Tamil Nadu, India; \\ mohanrajs.ece@mkce.ac.in, kavithav.ece@mkce.ac.in
}

\begin{abstract}
Objectives: To detect and remove the unwanted weeds present in an agricultural field and spray the pesticide at exact location of plants. Methods/Statistical Analysis: The entire process is implemented by using Labview programming with Machine Vision libraries. The movable agricultural vehicle is incorporated with machine vision library and microcontroller. The unwanted plants or weeds are identified by using Labview simulation process. Plants must be differentiated accordingly based on the type of weed that will affect the yield. The characteristics of weed should be properly studied. Findings: The unwanted plants or weeds grown will reduce the yield. Weed detection and removal in agricultural field is the main process. Utilization of the herbicides can be diminished just by appropriate breaking down of the weed. Application/Improvements: The position of weeds is automatically identified. Spraying of herbicide can be taken automatically or takes place once the position of the weed is detected by using spraying module.
\end{abstract}

Keywords: Herbicide Classification, Pattern Matching, Weed Detection

\section{Introduction}

In recent era, researchers showing more interest to develop agriculture based products for getting better yield. The work is mainly focused to detect weeds and spraying pesticides at exact location of herbicides. Expanded utilization of herbicide influences the well being of the humans and animals. Based on reported work, 33\% of loss is due to the unwanted weeds present in farm land which is shown in Figure 1. Farmland is not only affected by pests present in plant but also due to weeds in farmland which causes less productivity. So, it is needed to reduce losses. In India usage of herbicides are less when compared to other countries. Figure 2 shows the rate of pesticide used in India.

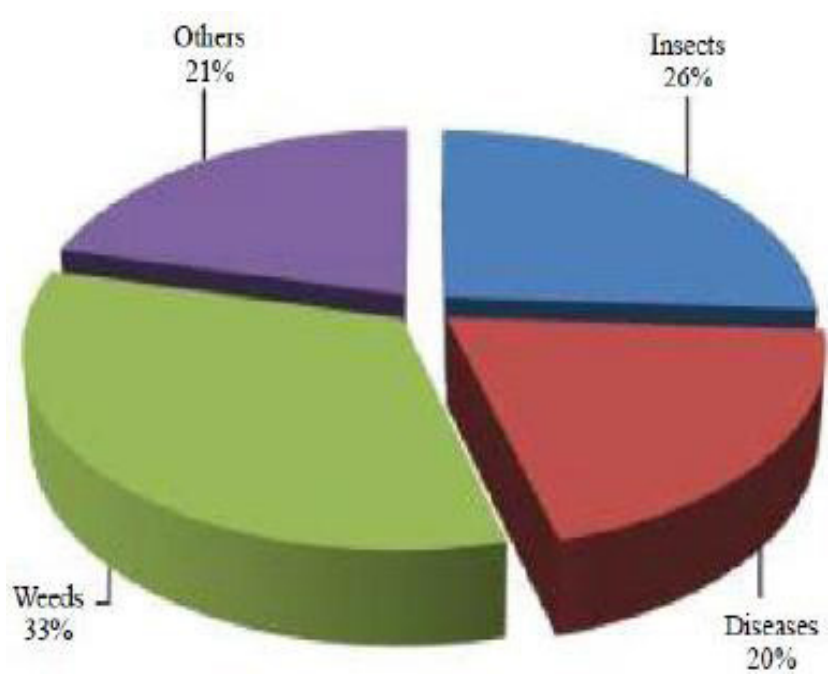

Figure 1. Crop yield caused by the weeds. 


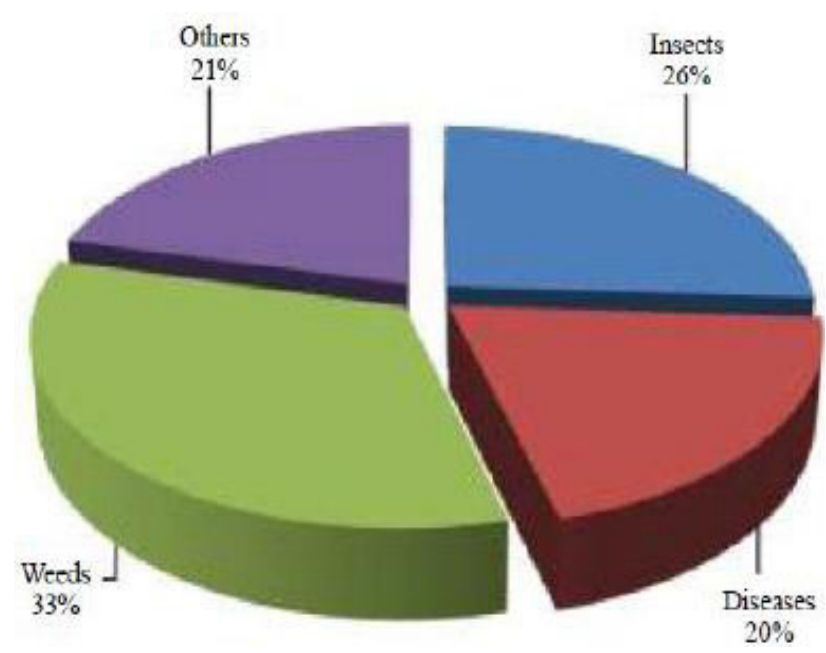

Figure 2. Usage of pesticide in Indian scenario.

Usage of herbicides in early stage can increase the yield and also reduces the waiting time for crop's growth. The procedures to control and eradicate the weed present in land require man power. There is a need to choose an alternative technique to reduce man power. This will create an opportunity to face this challenging world market.

Concept of wavelet based algorithm for weed detection is employed 1 . If the weed identification is not done properly, herbicide will be sprayed to other areas where there is no need of herbicides and it also contaminates water and soil. Image processing technique and wavelet based algorithm is used to classify the weeds.

This study ${ }^{2}$ relates the concept of automatic weed detection using image processing technique along with Matlab technique. The image processing technique helps to segregate weeds from normal cultivated plants and also it evaluates soil nutrition. This technique also helps to spray herbicide to the required environment and ensures the water contamination and soil pollution levels. This automatic machine vision based along with image processing technique is used to identify the weeds present in farm land. The agricultural field survey using image processing technique and machine vision system is shown in Figure 3.

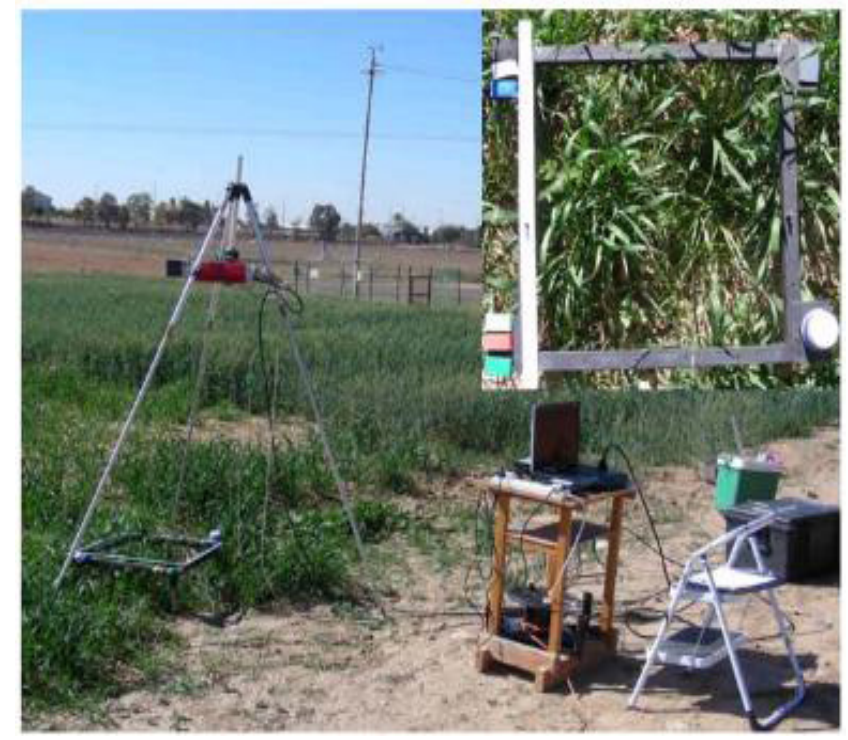

Figure 3. Surveying the field.

\section{Literature Survey}

There are three major models used to detect the weed in the agricultural land. They are discussed below.

\subsection{Biological MorphologyTechnique}

The biological morphology is used to predict the biological characteristics of a plant crop. The features include size and shape of the crop. With the help of the predicted size and shape of the plant captured we can easily identify whether the plant is a crop or a weed. Then to distinguish the plantation and soil proper segmentation algorithm is used based on the color of the image the image segmentation is performed. To reduce the noise present in the image proper filtering technique is used. Here median filter is used to remove the noise. By using the segmentation technique and median filtering $72.6 \%$ of accuracy is obtained. The preferred shape type is seven shape features to distinguish the crop and the weed and it is used in corn crop and obtained a result of $98.9 \%$. From the biological features it is easy to distinguish the soil and vegetation in an agricultural field $\underline{3-5}$. 


\subsection{Visual Texture Technique}

In visual texture system highlights of the photos for entropy, instance, vitality, differentiate, homogeneity, torpidity used for the plant recognizable proof also undesirable harvests. By using the estimation from guaranteeing backing vector Furthermore removing those organization includes got the precision of $93 \%$. Different research researchers about the weed discovery comes about give the diverse correctness's with different five surface highlights. The PCA with Gabor filter gives the precision accuracy about $91 \%$. The light intensity variation in the captured image used to find the weeds. Edge will be portrayed concerning representation sum from guaranteeing pixels accessible in the point of confinement of the region. The pixels appear in the images vary from zone to zone. Through the convergence of the pixel rate from the captured image at a particular zone is done again and again. It results the similarity of the weeds from the data set ${ }^{6,7}$.

\subsection{Automatic Spraying of Herbicide}

The main focus of this work is to identify the exact location of the weed and reduce the pesticide utilization and proper spraying. The sprayer utilized for the herbicide splashing might be the mechanical hand or engine or the sprinkler and so forth which is appeared in Figure 4. For requesting the weeds and yields in the rural field numerous creating calculation is utilized as a part of the current model. Different calculation procedures are accessible to distinguish the nearness of weeds from the harvests. The vast majority of the accessible procedures are finished utilizing picture preparing strategy ${ }^{\stackrel{8}{ }}$. For the most part it is utilized as a part of shading property, topological property, wavelet change, three wide band impedance channel yet the precision is less. In any case, the greater part of the weed identifying systems is displayed in the picture handling utilizing MATLAB ${ }^{9}$. In order to reduce the complexity in programming and hardware utilization Labview based machine vision system is introduced. Since it is an easy to understand method, accomplishes more noteworthy precision. The significant contrast between the

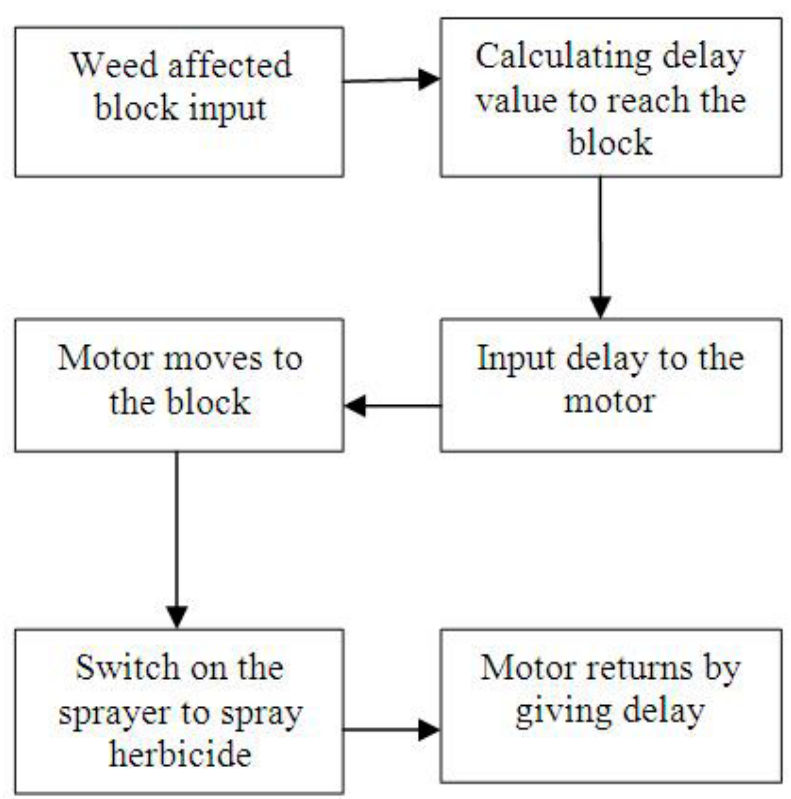

Figure 4. Working of the herbicide sprayer.

Table 1. Algorithm and its accuracy

\begin{tabular}{|c|c|}
\hline METHOD & ACCURACY (\%) \\
\hline $\begin{array}{c}\text { Spectral reflectance } \\
\text { property }\end{array}$ & $85-87 \%$ \\
\hline Color property & $50-96 \%$ \\
\hline Topology property & $83-91 \%$ \\
\hline Texture features & $30-78 \%$ \\
\hline Wavelet transform & $86-94 \%$ \\
\hline $\begin{array}{c}\text { Pattern matching } \\
\text { algorithm }\end{array}$ & $91-97 \%$ \\
\hline
\end{tabular}

current and the proposed method will gives the accurate results in the spraying unit as well as in the analyzing part in the field zones which is appeared in Table 1. Thus herbicide use will be diminished and it makes the ecofriendly idea of the farming field to procure more benefits and 
item result in limited ability to focus time in productive way $\underline{10}$.

\section{Proposed System}

The proposed framework will be included around three essential parts as appeared in Figure 5. As an issue of first significance will be picture acquirement that may be done by whatever sorts for cutting edge cameras for instance, to such an extent that regular webcams. The Polaroid should make acquainted peroxide blonde with ground. In this collection of confirmation, a camera module is interfaced

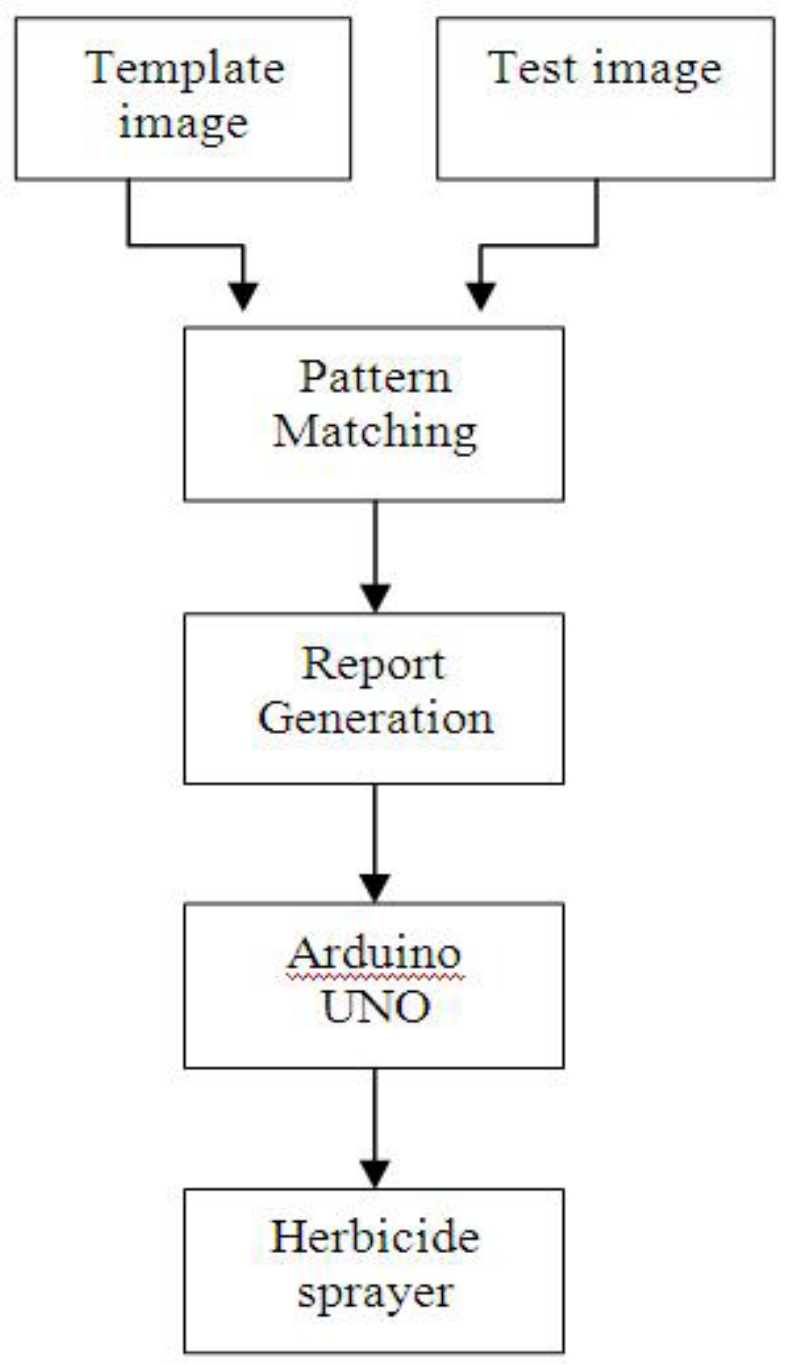

Figure 5. Working model of proposed system. with the herbicide sprayer at the height of 1.20 meters from the surface of the ground. The camera captures the picture of ground surface at $640 * 480$ pixels rate. The Labview tool is used to classify the weed and normal plant in the captured image by using machine vision algorithm. In the database the template images are loaded initially and they are trained. The camera captures the image and that image is considered as test image. The pattern matching algorithm is programmed in Labview tool. Now the test image is compared with the template images with the help of pattern matching algorithm. Based on the pattern the weed present in the test image is identified and the weed is classified from the normal plant. Based on the RGB colour difference the weed is identified with Labview programming. From the pattern matching results the report is generated and it is given to the Arduino UNO. The atmega328 microcontroller used in this work for getting accurate results and low power consumption. The sprayer unit is connected with the microcontroller, based on the result it will spray the herbicide only to the weed area. For each two minutes duration the picture is the field is taken which is used for further weed classification.

\section{Results and Discussion}

This study is centered on finding the weeds present in the field, automatically. By using the machine vision

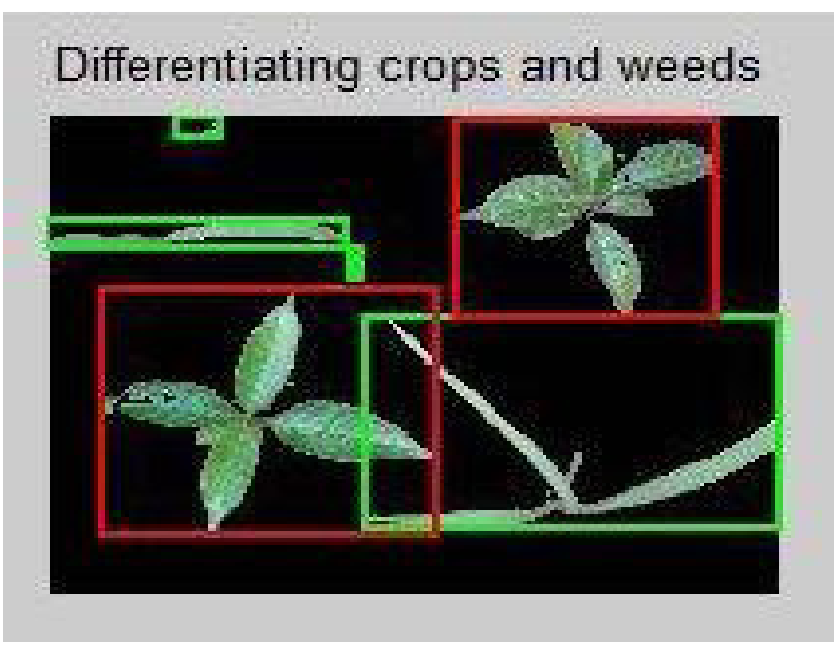

Figure 6. Experimental results. 
based algorithm with image processing 100 field images are selected as test images. The images are captured with different environmental conditions. From the 100 test images more than 91 percentage of the images are classified accurately. From the results the suggested technique performs better than other techniques. The red box in the Figure 6 indicates plants and green box indicates weeds.

\section{Conclusion}

The significant piece of this exactness condition is programmed horticultural weed location and showering of herbicide. Aside from these in this way limiting and keeping the pointless effects of the over use of substance herbicides and it lessens the required amount. This paper intends to display the new model of weed location and procurement of picture then at long last the weed characterization is finished by the example coordinating calculation and it was executed with Lab view and Adriano with machine vision. The significant preferred standpoint about the proposed framework when contrasted with the current calculation is arrangement exactness of $96 \%$.

\section{References}

1. Aware AA, Joshi K. Crop and weed detection based on texture and size features and automatic spraying of herbicides. International Journal of Advanced Research in Computer Science and Software Engineering. 2016; 6(1):1-7.

2. Malemath VS, Hugar SM. A new approach for weed detection in agriculture using image processing techniques.
International Journal of Advanced Scientific and Technical Research. 2016; 6(3):356-9.

3. Bhanumathi G, Subhakar B. Smart herbicide sprayer robot for agriculture fields. International Journal of Computer Science and Mobile Computing. 2015; 4(7):571-4.

4. Priyadharsini S, Sathiskumar BS. Developing a real time smart herbicide sprayer robot and automatic weed detection system. International Journal of Emerging Technology and Advanced Engineering. 2015; 5.

5. Shapira U, Herrmann I, Karnieli A, Bonfil JD. Weeds detection by ground level hyper spectral data. International Society for Photogrammetry and Remote Sensing. 201; 8(4):27-33.

6. Jones G, Truchetet F. Modeling agronomic images for weed detection and comparison of crop/weed discrimination algorithm performance. Precision Agriculture. 2009; 10(1):1-15. Crossref.

7. Piron A, Leemans V, Kleynen O, Lebeau F, Destain MF. Selection of the most efficient wavelength bands for discriminating weeds from crop. Computers and Electronics in Agriculture. 2008; 62:141-8. Crossref.

8. Sanchez A, Marchant A. Fusing 3D information forcrop/ weeds classification. Proceedings of the 15th International Conference on Pattern Recognition (ICPR'OO). 2000; 4:4295-99.

9. Samseemoung G, Soni P, Jayasuriya PWH, Salokhe MV. Application of Low Altitude Remote Sensing (LARS) platform for monitoring crop growth and weed infestation in a soyabean plantation. Precision Agriculture. 2012; 13(6):611-27. Crossref.

10. Xavier P, Burgos, Angela R, Alberto T, Gonzalo P, Cesar F. Improving weed pressure assessment using digital images from an experience-based reasoning approach. Computers and Electronics in Agriculture. 2009; 65:176-85. Crossref. 